\title{
Spectrum of the quadratic eigenparameter dependent discrete Dirac equations
}

\section{Turhan Koprubasi*}

\section{"Correspondence:}

tkoprubasi@kastamonu.edu.tr

Department of Mathematics, Kastamonu University, Kuzeykent,

Kastamonu, 37100, Turkey

\begin{abstract}
Let us consider the Boundary Value Problem (BVP) for the discrete Dirac equations $a_{n+1} y_{n+1}^{(2)}+b_{n} y_{n}^{(2)}+p_{n} y_{n}^{(1)}=\lambda y_{n}^{(1)}, a_{n-1} y_{n-1}^{(1)}+b_{n} y_{n}^{(1)}+a_{n} y_{n}^{(2)}=\lambda y_{n}^{(2)}, n \in \mathbb{N}$, $\left(\gamma_{0}+\gamma_{1} \lambda+\gamma_{2} \lambda^{2}\right) y_{1}^{(2)}+\left(\beta_{0}+\beta_{1} \lambda+\beta_{2} \lambda^{2}\right) y_{0}^{(1)}=0$, where $\left(a_{n}\right),\left(b_{n}\right),\left(p_{n}\right)$ and $\left(a_{n}\right), n \in \mathbb{N}$ are complex sequences, $\gamma_{i}, \beta_{i} \in \mathbb{C}, i=0,1,2$, and $\lambda$ is an eigenparameter. Discussing the eigenvalues and the spectral singularities, we prove that this BVP has a finite number of eigenvalues and spectral singularities with a finite number of multiplicities, if $\sum_{n=1}^{\infty} \exp \left(\varepsilon n^{\delta}\right)\left(\left|1-a_{n}\right|+\left|1+b_{n}\right|+\left|p_{n}\right|+\left|a_{n}\right|\right)<\infty$, holds, for some $\varepsilon>0$ and $\frac{1}{2} \leq \delta \leq 1$

MSC: 34L40; 47A10; 47A75
\end{abstract}

Keywords: discrete Dirac equations; eigenparameter; spectral analysis; discrete spectrum; spectral singularities

\section{Introduction}

In the last decade, discrete boundary value problems have been intensively studied. The spectral analysis of the difference equations have been treated by various authors in connection with the classical moment problem (see the monographs of Agarwal [1], AgarwalWong [2] and Kelley-Peterson [3] and the papers of Agarwal et al. [4, 5] and the references therein). Moreover, the modeling of certain linear and nonlinear problems in economies, optimal control theory, and other areas of study have led to the rapid development of the theory of difference equations. The spectral theory of the difference equations has also been applied to the solution of classes of nonlinear discrete Korteweg-de Vries equations and Toda lattices $[6,7]$.

Let us is consider the discrete boundary value problem (BVP)

$$
\begin{aligned}
& a_{n-1} y_{n-1}+b_{n} y_{n}+a_{n} y_{n+1}=\lambda y_{n}, \quad n \in \mathbb{N}=\{1,2, \ldots\}, \\
& y_{0}=0,
\end{aligned}
$$

where $\left(a_{n}\right)$ and $\left(b_{n}\right)$ are complex sequences, $a_{0} \neq 0$ and $\lambda$ is a spectral parameter. The spectral analysis of the BVP (1.1), (1.2) with continuous and point spectrum has been studied in [8]. In this article, the authors proved that the spectrum of the BVP (1.1), (1.2) consists of a continuous spectrum, eigenvalues and a spectral singularities. The spectral singularities are poles of the resolvent and are also imbedded in the continuous spectrum, but they are not eigenvalues. The effect of the spectral singularities in the spectral expansion of the BVP (1.1), (1.2) in terms of the principal vectors has been investigated in [9]. In [10, 
11], the dependence of the structure of the spectral singularities of the BVP (1.1), (1.2) on the behavior of the sequences $\left(a_{n}\right)$ and $\left(b_{n}\right)$ was considered. Some problems related to the spectral analysis of difference equations with spectral singularities have been discussed in [12-15]. The spectral analysis of an eigenparameter dependent non-selfadjoint BVP for the system of difference equations of first order has been studied in $[16,17]$.

Let us consider the non-selfadjoint BVP for the system of difference equations of first order

$$
\begin{aligned}
& \left\{\begin{array}{l}
a_{n+1} y_{n+1}^{(2)}+b_{n} y_{n}^{(2)}+p_{n} y_{n}^{(1)}=\lambda y_{n}^{(1)}, \\
a_{n-1} y_{n-1}^{(1)}+b_{n} y_{n}^{(1)}+q_{n} y_{n}^{(2)}=\lambda y_{n}^{(2)}, \quad n \in \mathbb{N},
\end{array}\right. \\
& \left(\gamma_{0}+\gamma_{1} \lambda+\gamma_{2} \lambda^{2}\right) y_{1}^{(2)}+\left(\beta_{0}+\beta_{1} \lambda+\beta_{2} \lambda^{2}\right) y_{0}^{(1)}=0,
\end{aligned}
$$

where $\left(\begin{array}{c}y_{n}^{(1)} \\ y_{n}^{(2)}\end{array}\right), n \in \mathbb{N}$ are vector sequences, $a_{n} \neq 0, b_{n} \neq 0$ for all $n$. Also $\gamma_{2} \neq \frac{-\beta_{1}}{a_{0}},\left|\gamma_{2}\right|+\left|\beta_{2}\right| \neq$ 0 , and $\gamma_{0} \beta_{1}-\gamma_{1} \beta_{0} \neq 0$ where $\gamma_{i}, \beta_{i} \in \mathbb{C}, i=0,1,2$. If $a_{n} \equiv 1$ and $b_{n} \equiv-1$ for all $n \in \mathbb{N}$, then the system (1.3) reduces to

$$
\left\{\begin{array}{l}
\Delta y_{n}^{(2)}+p_{n} y_{n}^{(1)}=\lambda y_{n}^{(1)}, \\
-\Delta y_{n-1}^{(1)}+q_{n} y_{n}^{(2)}=\lambda y_{n}^{(2)}, \quad n \in \mathbb{N},
\end{array}\right.
$$

where $\Delta$ is a forward difference operator. The system (1.5) is the discrete analog of the well-known Dirac system

$$
\left(\begin{array}{cc}
0 & 1 \\
-1 & 0
\end{array}\right)\left(\begin{array}{l}
y_{1}^{\prime} \\
y_{2}^{\prime}
\end{array}\right)+\left(\begin{array}{cc}
p(x) & 0 \\
0 & q(x)
\end{array}\right)\left(\begin{array}{l}
y_{1} \\
y_{2}
\end{array}\right)=\lambda\left(\begin{array}{l}
y_{1} \\
y_{2}
\end{array}\right)
$$

(see [18], Chapter 2). Therefore the system (1.5) (also (1.3)) is called a discrete Dirac system. The specific feature of this paper is the presence of the spectral parameter not only in the difference equation and also in the boundary condition, which is quadratic.

In this article, we intend to investigate eigenvalues and spectral singularities of the BVP (1.3), (1.4), which has a finite number of eigenvalues and spectral singularities with a finite number of multiplicities, if the condition

$$
\sum_{n=1}^{\infty} \exp \left(\varepsilon n^{\delta}\right)\left(\left|1-a_{n}\right|+\left|1+b_{n}\right|+\left|p_{n}\right|+\left|q_{n}\right|\right)<\infty
$$

holds, for some $\varepsilon>0$ and $\frac{1}{2} \leq \delta \leq 1$.

\section{Jost solution of (1.3)}

Suppose that the condition

$$
\sum_{n=1}^{\infty} \exp \left(\varepsilon n^{\delta}\right)\left(\left|1-a_{n}\right|+\left|1+b_{n}\right|+\left|p_{n}\right|+\left|q_{n}\right|\right)<\infty
$$

is satisfied for some $\varepsilon>0$ and $\frac{1}{2} \leq \delta \leq 1$. It is well known [15] that (1.3) has the bounded solution

$$
f_{n}(z)=\left(\begin{array}{l}
f_{n}^{(1)}(z) \\
f_{n}^{(2)}(z)
\end{array}\right)=\alpha_{n}\left(I_{2}+\sum_{m=1}^{\infty} A_{n m} e^{i m z}\right)\left(\begin{array}{c}
e^{i \frac{z}{2}} \\
-i
\end{array}\right) e^{i n z}, \quad n \in \mathbb{N},
$$




$$
f_{0}^{(1)}(z)=\alpha_{0}^{11}\left\{e^{i \frac{z}{2}}\left[1+\sum_{m=1}^{\infty} A_{0 m}^{11} e^{i m z}\right]-i \sum_{m=1}^{\infty} A_{0 m}^{12} e^{i m z}\right\}
$$

under the condition (2.1) for $\lambda=2 \sin \frac{z}{2}$ and $z \in \overline{\mathbb{C}}_{+}:=\{z: z \in \mathbb{C}, \operatorname{Im} z \geq 0\}$, where

$$
\alpha_{n}=\left(\begin{array}{cc}
\alpha_{n}^{11} & \alpha_{n}^{12} \\
\alpha_{n}^{21} & \alpha_{n}^{22}
\end{array}\right), \quad I_{2}=\left(\begin{array}{cc}
1 & 0 \\
0 & 1
\end{array}\right), \quad A_{n m}=\left(\begin{array}{ll}
A_{n m}^{11} & A_{n m}^{12} \\
A_{n m}^{21} & A_{n m}^{22}
\end{array}\right) .
$$

Note that $\alpha_{n}^{i j}$ and $A_{n m}^{i j}(i, j=1,2)$ are expressed in terms of $\left(a_{n}\right),\left(b_{n}\right),\left(p_{n}\right)$, and $\left(q_{n}\right), n \in \mathbb{N}$. Also

$$
\left|A_{n m}^{i j}\right| \leq C \sum_{k=n+\left[\left|\frac{m}{2}\right|\right]}^{\infty}\left(\left|1-a_{k}\right|+\left|1+b_{k}\right|+\left|p_{k}\right|+\left|q_{k}\right|\right)
$$

holds, where $C>0$ is a constant and $\left[\left|\frac{m}{2}\right|\right]$ is the integer part of $\frac{m}{2}$. Therefore $f_{n}$ is a vectorvalued analytic function with respect to $z$ in $\mathbb{C}_{+}:=\{z: z \in \mathbb{C}, \operatorname{Im} z>0\}$ and continuous in $\overline{\mathbb{C}}_{+}[15]$. The solution $f(z)=\left(f_{n}(z)\right)=\left(\begin{array}{l}f_{n}^{(1)}(z) \\ f_{n}^{(2)}(z)\end{array}\right)$ is called the Jost solution of (1.3).

\section{Eigenvalues and spectral singularities of (1.3)-(1.4)}

We define

$$
\begin{aligned}
f(z)= & \left(\gamma_{0}+2 \gamma_{1} \sin \frac{z}{2}+4 \gamma_{2} \sin ^{2} \frac{z}{2}\right) f_{1}^{(2)}(z) \\
& +\left(\beta_{0}+2 \beta_{1} \sin \frac{z}{2}+4 \beta_{2} \sin ^{2} \frac{z}{2}\right) f_{0}^{(1)}(z)
\end{aligned}
$$

using (1.4), (2.2), and (2.3). It follows from (2.2) and (2.3) that the function $f$ is analytic in $\mathbb{C}_{+}$, continuous up to the real axis and

$$
f(z+4 \pi)=f(z)
$$

Let

$$
T_{0}:=\{z: z \in \mathbb{C}, z=x+i y,-2 \pi \leq x \leq 2 \pi, y>0\}, \quad T:=T_{0} \cup[-2 \pi, 2 \pi] .
$$

We will denote the set of all eigenvalues and spectral singularities of the BVP (1.3), (1.4) by $\sigma_{d}$ and $\sigma_{s s}$, respectively. It is clear that

$$
\begin{aligned}
& \sigma_{d}=\left\{\lambda: \lambda=2 \sin \frac{z}{2}, z \in T_{0}, f(z)=0\right\}, \\
& \sigma_{s s}=\left\{\lambda: \lambda=2 \sin \frac{z}{2}, z \in[-2 \pi, 2 \pi], f(z)=0\right\} .
\end{aligned}
$$

From (2.2), (2.3), and (3.1) we obtain

$$
\begin{aligned}
f(z)= & -\alpha_{0}^{11} \beta_{2} e^{-i \frac{z}{2}}+i\left(\alpha_{1}^{22} \gamma_{2}+\alpha_{0}^{11} \beta_{1}\right) \\
& +\left[\alpha_{1}^{22} \gamma_{1}-\alpha_{1}^{21} \gamma_{2}+\alpha_{0}^{11}\left(\beta_{0}+2 \beta_{2}\right)\right] e^{i \frac{z}{2}}
\end{aligned}
$$




$$
\begin{aligned}
& +i\left[-\alpha_{1}^{22}\left(\gamma_{0}+2 \gamma_{2}\right)+\alpha_{1}^{21} \gamma_{1}-\alpha_{0}^{11} \beta_{1}\right] e^{i z} \\
& +\left[-\alpha_{1}^{22} \gamma_{1}+\alpha_{1}^{21}\left(\gamma_{0}+2 \gamma_{2}\right)-\alpha_{0}^{11} \beta_{2}\right] e^{i \frac{3 z}{2}} \\
& +i\left(\alpha_{1}^{22} \gamma_{2}-\alpha_{1}^{21} \gamma_{1}\right) e^{2 i z}-\alpha_{1}^{21} \gamma_{2} e^{i \frac{5 z}{2}} \\
& +i \sum_{m=1}^{\infty} \alpha_{0}^{11} \beta_{2} A_{0 m}^{12} e^{i(m-1) z}+\sum_{m=1}^{\infty} \alpha_{0}^{11}\left(\beta_{1} A_{0 m}^{12}-\beta_{2} A_{0 m}^{11}\right) e^{i\left(m-\frac{1}{2}\right) z} \\
& +i \sum_{m=1}^{\infty}\left\{\gamma_{2}\left(\alpha_{1}^{22} A_{1 m}^{22}+\alpha_{1}^{21} A_{1 m}^{12}\right)\right. \\
& \left.+\alpha_{0}^{11}\left[\beta_{1} A_{0 m}^{11}-\left(\beta_{0}+2 \beta_{2}\right) A_{0 m}^{12}\right]\right\} e^{i m z} \\
& +\sum_{m=1}^{\infty}\left\{\alpha_{1}^{22}\left(\gamma_{1} A_{1 m}^{22}-\gamma_{2} A_{1 m}^{21}\right)+\alpha_{1}^{21}\left(\gamma_{1} A_{1 m}^{12}-\gamma_{2} A_{1 m}^{11}\right)\right. \\
& \left.+\alpha_{0}^{11}\left[\left(\beta_{0}+2 \beta_{2}\right) A_{0 m}^{11}-\beta_{1} A_{0 m}^{12}\right]\right\} e^{i\left(m+\frac{1}{2}\right) z} \\
& +i \sum_{m=1}^{\infty}\left\{-\alpha_{1}^{22}\left[\left(\gamma_{0}+2 \gamma_{2}\right) A_{1 m}^{22}-\gamma_{1} A_{1 m}^{21}\right]\right. \\
& -\alpha_{1}^{21}\left[\left(\gamma_{0}+2 \gamma_{2}\right) A_{1 m}^{12}-\gamma_{1} A_{1 m}^{11}\right] \\
& \left.+\alpha_{0}^{11}\left(\beta_{2} A_{0 m}^{12}-\beta_{1} A_{0 m}^{11}\right)\right\} e^{i(m+1) z} \\
& +\sum_{m=1}^{\infty}\left\{-\alpha_{1}^{22}\left[\gamma_{1} A_{1 m}^{22}-\left(\gamma_{0}+2 \gamma_{2}\right) A_{1 m}^{21}\right]\right. \\
& +\alpha_{1}^{21}\left[\left(\gamma_{0}+2 \gamma_{2}\right) A_{1 m}^{11}-\gamma_{1} A_{1 m}^{12}\right] \\
& \left.-\alpha_{0}^{11} \beta_{2} A_{0 m}^{11}\right\} e^{i\left(m+\frac{3}{2}\right) z} \\
& +i \sum_{m=1}^{\infty}\left[\alpha_{1}^{22}\left(\gamma_{2} A_{1 m}^{22}-\gamma_{1} A_{1 m}^{21}\right)+\alpha_{1}^{21}\left(\gamma_{2} A_{1 m}^{12}-\gamma_{1} A_{1 m}^{11}\right)\right] e^{i(m+2) z} \\
& +\sum_{m=1}^{\infty}-\gamma_{2}\left(\alpha_{1}^{22} A_{1 m}^{21}+\alpha_{1}^{21} \gamma_{2} A_{1 m}^{11}\right) e^{i\left(m+\frac{5}{2}\right) z}
\end{aligned}
$$

Let

$$
F(z):=f(z) e^{i \frac{z}{2}}
$$

then the function $F$ is analytic in $\mathbb{C}_{+}$, continuous in $\overline{\mathbb{C}}_{+}$,

$$
\begin{aligned}
F(z)= & -\alpha_{0}^{11} \beta_{2}+i\left(\alpha_{1}^{22} \gamma_{2}+\alpha_{0}^{11} \beta_{1}\right) e^{i \frac{z}{2}} \\
& +\left[\alpha_{1}^{22} \gamma_{1}-\alpha_{1}^{21} \gamma_{2}+\alpha_{0}^{11}\left(\beta_{0}+2 \beta_{2}\right)\right] e^{i z} \\
& +i\left[-\alpha_{1}^{22}\left(\gamma_{0}+2 \gamma_{2}\right)+\alpha_{1}^{21} \gamma_{1}-\alpha_{0}^{11} \beta_{1}\right] e^{i \frac{3 z}{2}} \\
& +\left[-\alpha_{1}^{22} \gamma_{1}+\alpha_{1}^{21}\left(\gamma_{0}+2 \gamma_{2}\right)-\alpha_{0}^{11} \beta_{2}\right] e^{2 i z} \\
& +i\left(\alpha_{1}^{22} \gamma_{2}-\alpha_{1}^{21} \gamma_{1}\right) e^{i \frac{5 z}{2}}-\alpha_{1}^{21} \gamma_{2} e^{3 i z} \\
& +i \sum_{m=1}^{\infty} \alpha_{0}^{11} \beta_{2} A_{0 m}^{12} e^{i\left(m-\frac{1}{2}\right) z}+\sum_{m=1}^{\infty} \alpha_{0}^{11}\left(\beta_{1} A_{0 m}^{12}-\beta_{2} A_{0 m}^{11}\right) e^{i m z}
\end{aligned}
$$




$$
\begin{aligned}
& +i \sum_{m=1}^{\infty}\left\{\gamma_{2}\left(\alpha_{1}^{22} A_{1 m}^{22}+\alpha_{1}^{21} A_{1 m}^{12}\right)\right. \\
& \left.+\alpha_{0}^{11}\left[\beta_{1} A_{0 m}^{11}-\left(\beta_{0}+2 \beta_{2}\right) A_{0 m}^{12}\right]\right\} e^{i\left(m+\frac{1}{2}\right) z} \\
& +\sum_{m=1}^{\infty}\left\{\alpha_{1}^{22}\left(\gamma_{1} A_{1 m}^{22}-\gamma_{2} A_{1 m}^{21}\right)+\alpha_{1}^{21}\left(\gamma_{1} A_{1 m}^{12}-\gamma_{2} A_{1 m}^{11}\right)\right. \\
& \left.+\alpha_{0}^{11}\left[\left(\beta_{0}+2 \beta_{2}\right) A_{0 m}^{11}-\beta_{1} A_{0 m}^{12}\right]\right\} e^{i(m+1) z} \\
& +i \sum_{m=1}^{\infty}\left\{-\alpha_{1}^{22}\left[\left(\gamma_{0}+2 \gamma_{2}\right) A_{1 m}^{22}-\gamma_{1} A_{1 m}^{21}\right]\right. \\
& -\alpha_{1}^{21}\left[\left(\gamma_{0}+2 \gamma_{2}\right) A_{1 m}^{12}-\gamma_{1} A_{1 m}^{11}\right] \\
& \left.+\alpha_{0}^{11}\left(\beta_{2} A_{0 m}^{12}-\beta_{1} A_{0 m}^{11}\right)\right\} e^{i\left(m+\frac{3}{2}\right) z} \\
& +\sum_{m=1}^{\infty}\left\{-\alpha_{1}^{22}\left[\gamma_{1} A_{1 m}^{22}-\left(\gamma_{0}+2 \gamma_{2}\right) A_{1 m}^{21}\right]\right. \\
& +\alpha_{1}^{21}\left[\left(\gamma_{0}+2 \gamma_{2}\right) A_{1 m}^{11}-\gamma_{1} A_{1 m}^{12}\right] \\
& \left.-\alpha_{0}^{11} \beta_{2} A_{0 m}^{11}\right\} e^{i(m+2) z} \\
& +i \sum_{m=1}^{\infty}\left[\alpha_{1}^{22}\left(\gamma_{2} A_{1 m}^{22}-\gamma_{1} A_{1 m}^{21}\right)+\alpha_{1}^{21}\left(\gamma_{2} A_{1 m}^{12}-\gamma_{1} A_{1 m}^{11}\right)\right] e^{i\left(m+\frac{5}{2}\right) z} \\
& +\sum_{m=1}^{\infty}-\gamma_{2}\left(\alpha_{1}^{22} A_{1 m}^{21}+\alpha_{1}^{21} \gamma_{2} A_{1 m}^{11}\right) e^{i(m+3) z}
\end{aligned}
$$

and

$$
F(z+4 \pi)=F(z)
$$

Using (3.2)-(3.5),

$$
\begin{aligned}
& \sigma_{d}=\left\{\lambda: \lambda=2 \sin \frac{z}{2}, z \in T_{0}, F(z)=0\right\}, \\
& \sigma_{s s}=\left\{\lambda: \lambda=2 \sin \frac{z}{2}, z \in[-2 \pi, 2 \pi], F(z)=0\right\} .
\end{aligned}
$$

Definition 3.1 The multiplicity of a zero of $F$ in $T$ is called the multiplicity of the corresponding eigenvalue or spectral singularity of the BVP (1.3), (1.4).

It follows from (3.2) and (3.3) that, in order to investigate the quantitative properties of the eigenvalues and the spectral singularities of the BVP (1.3), (1.4), we need to discuss the quantitative properties of the zeros of $F$ in $T$.

Let

$$
\begin{aligned}
& S_{1}:=\left\{z: z \in T_{0}, F(z)=0\right\}, \\
& S_{2}:=\{z: z \in[-2 \pi, 2 \pi], F(z)=0\} .
\end{aligned}
$$

We also denote the set of all limit points of $S_{1}$ by $S_{3}$ and the set of all zeros of $F$ with infinite multiplicity by $S_{4}$. 
From (3.2), (3.3), and (3.9) we get

$$
\begin{aligned}
& \sigma_{d}=\left\{\lambda: \lambda=2 \sin \frac{z}{2}, z \in S_{1}\right\}, \\
& \sigma_{s s}=\left\{\lambda: \lambda=2 \sin \frac{z}{2}, z \in S_{2}\right\} .
\end{aligned}
$$

Theorem 3.1 If (2.1) holds, then:

(i) The set $S_{1}$ is bounded and countable.

(ii) $S_{1} \cap S_{3}=\varnothing, S_{1} \cap S_{4}=\varnothing$.

(iii) The set $S_{2}$ is compact and $\mu\left(S_{2}\right)=0$, where $\mu$ denotes the Lebesgue measure in the real axis.

(iv) $S_{3} \subset S_{2}, S_{4} \subset S_{2} ; \mu\left(S_{3}\right)=\mu\left(S_{4}\right)=0$.

(v) $S_{3} \subset S_{4}$.

Proof Using (1.4), (2.4), and (3.6), we have

$$
F(z)= \begin{cases}-\alpha_{0}^{11} \beta_{2}+o\left(e^{-y}\right), & \beta_{2} \neq 0, z \in T, y \rightarrow \infty \\ i\left(\alpha_{1}^{22} \gamma_{2}+\alpha_{0}^{11} \beta_{1}\right) e^{i \frac{z}{2}}+o\left(e^{-y}\right), & \beta_{2}=0, z \in T, y \rightarrow \infty\end{cases}
$$

Equation (3.11) shows that $S_{1}$ is bounded. Since $F$ is analytic in $\mathbb{C}_{+}$and is a $4 \pi$ periodic function, we find that $S_{1}$ has at most a countable number of elements. This proves (i).

From the uniqueness theorems of analytic functions we obtain (ii)-(iv) [19].

Using the continuity of all derivatives of $F$ on $[-2 \pi, 2 \pi]$ we get (v).

From (3.10) and Theorem 3.1, we have the following.

Theorem 3.2 Under the condition (2.1):

(i) The set of eigenvalues of the BVP (1.3), (1.4) is bounded and countable and its limit points can lie only in $[-2,2]$.

(ii) $\sigma_{s s} \subset[-2,2], \sigma_{s s}=\bar{\sigma}_{s s}$ and $\mu\left(\sigma_{s s}\right)=0$.

For $\delta=1$ the condition (2.1) reduces to

$$
\sum_{n=1}^{\infty} \exp (\varepsilon n)\left(\left|1-a_{n}\right|+\left|1+b_{n}\right|+\left|p_{n}\right|+\left|q_{n}\right|\right)<\infty
$$

Theorem 3.3 Under the condition (3.12) the BVP (1.3), (1.4) has a finite number of eigenvalues and spectral singularities and each of them is of finite multiplicity.

Proof Using (2.4) we find that

$$
\left|A_{n m}^{i j}\right| \leq C \exp \left[-\frac{\varepsilon}{4}(n+m)\right], \quad i, j=1,2, n, m \in \mathbb{N}
$$

where $C>0$ is a constant. From (3.6) and (3.13) we observe that the function $F$ has an analytic continuation to the half-plane $\operatorname{Im} z>-\frac{\varepsilon}{4}$. Since $F$ is a $4 \pi$ periodic function, the limit points its zeros in $T$ cannot lie in $[-2 \pi, 2 \pi]$. Using Theorem 3.1 we have the bounded 
sets $S_{1}$ and $S_{2}$ having a finite number of elements. From analyticity of $F$ in $\operatorname{Im} z>-\frac{\varepsilon}{4}$, we find that all zeros of $F$ in $T$ have a finite multiplicity. Therefore using (3.10), we obtain the finiteness of the eigenvalues and spectral singularities of the BVP (1.3), (1.4).

It is seen that the condition (3.12) guarantees the analytic continuation of $F$ from the real axis to lower half-plane. So the finiteness of eigenvalues and spectral singularities of the BVP (1.3), (1.4) are obtained as a result of this analytic continuation.

Now let us suppose that

$$
\sum_{n=1}^{\infty} \exp \left(\varepsilon n^{\delta}\right)\left(\left|1-a_{n}\right|+\left|1+b_{n}\right|+\left|p_{n}\right|+\left|q_{n}\right|\right)<\infty, \quad \varepsilon>0, \frac{1}{2} \leq \delta<1,
$$

which is weaker than (3.12). It is evident that under the condition (3.14) the function $F$ is analytic in $\mathbb{C}_{+}$and infinitely differentiable on the real axis. But $F$ does not have an analytic continuation from the real axis to lower half-plane. Therefore under the condition (3.14) the finiteness of eigenvalues and spectral singularities of the BVP (1.3), (1.4) cannot be shown in a way similar to Theorem 3.3.

Under the condition (3.14), to prove that the eigenvalues and the spectral singularities of the BVP (1.3), (1.4) are of finite number we will use the following.

Theorem 3.4 ([14]) Let us assume that the $4 \pi$ periodic function $g$ is analytic in $\mathbb{C}_{+}$, all of its derivatives are continuous in $\overline{\mathbb{C}}_{+}$and

$$
\sup _{z \in T}\left|g^{(k)}(z)\right| \leq A_{k}, \quad k \in \mathbb{N} \cup\{0\}
$$

If the set $G \subset[-2 \pi, 2 \pi]$ with Lebesgue measure zero is the set of all zeros the function $g$ with infinite multiplicity in $T$, if

$$
\int_{0}^{\omega} \ln K(s) d \mu\left(G_{s}\right)=-\infty
$$

where $K(s)=\inf _{k} \frac{A_{k} s^{k}}{k !}$ and $\mu\left(G_{s}\right)$ is the Lebesgue measure of s-neighborhood of $G$ and $\omega \in$ $(-2 \pi, 2 \pi)$ is an arbitrary constant, then $g \equiv 0$ in $\overline{\mathbb{C}}_{+}$.

Under the condition (3.14) from (2.4) and (3.6) we find

$$
\left|F^{(k)}(z)\right| \leq A_{k}, \quad k \in \mathbb{N} \cup\{0\},
$$

where

$$
A_{k}=2^{k} C \sum_{m=1}^{\infty} m^{k} \exp \left(-\varepsilon m^{\delta}\right)
$$

and $C>0$ is a constant. We can obtain the following estimate:

$$
A_{k} \leq 2^{k} C \int_{0}^{\infty} x^{k} \exp \left(-\varepsilon x^{\delta}\right) d x \leq D d^{k} k ! k^{k \frac{1-\delta}{\delta}},
$$

where $D$ and $d$ are constants depending on $C, \varepsilon$, and $\delta$. 
Theorem 3.5 If (3.14) holds, then $S_{4}=\varnothing$.

Proof The function $F$ satisfies all conditions of Theorem 3.4 except (3.15). But $F$ is not identically equal to zero. In this case the function $F$ satisfies the condition

$$
\int_{0}^{\omega} \ln K(s) d \mu\left(S_{4, s}\right)>-\infty
$$

instead of (3.15), where $K(s)=\inf _{k} \frac{A_{k} s^{k}}{k !}, k \in \mathbb{N} \cup\{0\}$, and $\mu\left(S_{4, s}\right)$ is the Lebesgue measure of the $s$-neighborhood of $S_{4}$ and $A_{k}$ defined by (3.16). Substituting (3.16) in the definition of $K(s)$, we get

$$
K(s)=D \exp \left\{-\frac{1-\delta}{\delta} e^{-1} d^{-\frac{\delta}{1-\delta}} s^{-\frac{\delta}{1-\delta}}\right\} .
$$

It follows from (3.17) and (3.18) that

$$
\int_{0}^{\omega} s^{-\frac{\delta}{1-\delta}} d \mu\left(S_{4, s}\right)<\infty
$$

Since $\frac{\delta}{1-\delta} \geq 1$, (3.19) holds for arbitrary $s$ if and only if $\mu\left(S_{4, s}\right)=0$ or $S_{4}=\varnothing$.

Theorem 3.6 Under the condition (3.14) the BVP (1.3), (1.4) has a finite number of eigenvalues and spectral singularities and each of them is of finite multiplicity.

Proof To be able to prove the theorem we have to show that the function $F$ has a finite number of zeros with finite multiplicities in $T$.

From Theorem 3.1 and Theorem 3.5 we see that $S_{3}=\varnothing$. So the bounded sets $S_{1}$ and $S_{2}$ have no limit points, i.e., the function $F$ has only a finite number of zeros in $T$. Since $S_{4}=\varnothing$, these zeros are of finite multiplicity.

\section{Competing interests}

The author declares that they have no competing interests.

Received: 26 February 2014 Accepted: 5 May 2014 Published: 16 May 2014

\section{References}

1. Agarwal, RP: Difference Equation and Inequalities: Theory, Methods and Applications. Dekker, New York (2000)

2. Agarwal, RP, Wong, PJY: Advanced Topics in Difference Equations. Kluwer Academic, Dordrecht (1997)

3. Kelley, WG, Peterson, AC: Difference Equations: An Introduction with Applications. Academic Press, San Diego (2001)

4. Agarwal, RP, Perera, K, O'Regan, D: Multiple positive solutions of singular and nonsingular discrete problems via variational methods. Nonlinear Anal. 58, 69-73 (2004)

5. Agarwal, RP, Perera, K, O'Regan, D: Multiple positive solutions of singular discrete $p$-Laplacian problems via variational methods. Adv. Differ. Equ. 2005(2), 93-99 (2005)

6. Berezanski, YM: Integration of nonlinear difference equations by the inverse spectral problem method. Sov. Math. Dokl. 31, 264-267 (1985)

7. Toda, M: Theory of Nonlinear Lattices. Springer, Berlin (1981)

8. Bairamov, E, Cakar, O, Krall, AM: Non-selfadjoint difference operators and Jacobi matrices with spectral singularities. Math. Nachr. 229, 5-14 (2001)

9. Krall, AM, Bairamov, E, Cakar, O: Spectral analysis of non-selfadjoint discrete Schrödinger operator with spectral singularities. Math. Nachr. 231, 89-104 (2001)

10. Adivar, M, Bairamov, E: Spectral properties of non-selfadjoint difference operators. J. Math. Anal. Appl. 261, 461-478 (2001)

11. Adivar, M, Bairamov, E: Difference equations of second order with spectral singularities. J. Math. Anal. Appl. 277 714-721 (2003) 
12. Adivar, M, Bohner, M: Spectral analysis of $q$-difference equations with spectral singularities. Math. Comput. Model. 43(7-9), 695-703 (2006)

13. Adivar, M, Bohner, M: Spectrum and principal vectors of second order $q$-difference equations. Indian J. Math. 48(1), 17-33 (2006)

14. Bairamov, E, Celebi, AO: Spectrum and spectral expansion for the non-selfadjoint discrete Dirac operators. Q. J. Math. 50(2), 371-384 (1999)

15. Bairamov, E, Coskun, C: Jost solutions and the spectrum of the system of difference equations. Appl. Math. Lett. 17, 1039-1045 (2004)

16. Bairamov, E, Koprubasi, T: Eigenparameter dependent discrete Dirac equations with spectral singularities. Appl. Math. Comput. 215, 4216-4220 (2010)

17. Aygar, Y, Olgun, M, Koprubasi, T: Principal functions of nonselfadjoint discrete Dirac equations with spectral parameter in boundary conditions. Abstr. Appl. Anal. 2012, Article ID 924628 (2012)

18. Levitan, BM, Sargsjan, IS: Introduction to Spectral Theory. Translations of Mathematical Monographs, vol. 39 (1975)

19. Dolzhenko, EP: Boundary value uniqueness theorems for analytic functions. Math. Notes 26(6), 437-442 (1979)

10.1186/1687-1847-2014-148

Cite this article as: Koprubasi: Spectrum of the quadratic eigenparameter dependent discrete Dirac equations. Advances in Difference Equations 2014, 2014:148

\section{Submit your manuscript to a SpringerOpen ${ }^{\circ}$ journal and benefit from:}

- Convenient online submission

- Rigorous peer review

- Immediate publication on acceptance

- Open access: articles freely available online

- High visibility within the field

- Retaining the copyright to your article 